\title{
DESIGN OF INDOOR ROBOT PROTOTYPE GUIDED BY RFID BASED POSITIONING AND NAVIGATION SYSTEM
}

\author{
Emrullah Demiral $^{1 *}$, İsmail Rakıp Karaş ${ }^{1, \text { a }}$, Yusuf Karakaya ${ }^{1}$, Mykola Kozlenko $^{2}$ \\ ${ }^{1}$ Dept. of Computer Engineering Karabuk University, Karabuk, Turkey - (ismailkaras, emrullahdemiral)@karabuk.edu.tr, \\ karakayayusuf18@gmail.com) \\ ${ }^{2}$ Vasyl Stefanyk Precarpathian National University, Ivano-Frankivsk, Ukraine - (mykola.kozlenko@pnu.edu.ua)
}

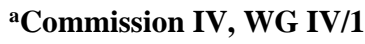

KEY WORDS: Indoor robot, RFID based Indoor Location System, Indoor Navigation, Alphabot, Arduino

\begin{abstract}
:
In this study, a robot prototype was designed for indoor spaces guided by an RFID-based positioning and navigation system. First, the work area was prepared from cardboard material and RFID cards were placed at predetermined points in the work area. The unique ID number of each RFID card was defined and the coordinates of their location in the work area are known. The RFID reader in the robot prototype reads from less than $5 \mathrm{~cm}$. With a basic approach, when the robot reads an RFID card that it passes over while in motion, the position of the robot is considered the same as the position of the card it is currently reading. The route is defined for the robot prototype whose location is known before starting the movement. When the robot reads a new RFID card during movement, it must move forward or turn left or right to reach the point where the next RFID card is located according to the route. This decision was predetermined and defined according to its location. Alphabot was used as the prototype. Arduino board and additional auxiliary sensors such as gyro sensor, speed sensors, distance sensors are placed on the prototype. The prototype robot is left at any point in the work area and arrives at a target point determined by the user. The required road route to reach the destination is calculated with the shortest path algorithm depending on the road network on the working area and the route is defined. Thus, it is ensured that the prototype reaches the target without any external intervention by the user other than target determination.
\end{abstract}

\section{INTRODUCTION}

Since the first day of existence, human beings are constantly in search of both surviving and living life more easily. Consequently, it designs and develops tools to be used for various purposes. With these searches that lasted for ages, technology has developed and continues to develop. As a result of this development, human beings are now developing autonomous robots that do not use human power. These robots save both power and time.

In today's modern cities, the number of multi-storey and complex buildings is increasing day by day. The fact that the buildings are large, complex and crowded brings with it many new problems waiting to be solved. In emergencies that may occur, the need to intervene with the disaster victims in the building as soon as possible is the foremost of these problems (Albert and Henry 2009). Apart from this, processes such as indoor navigation, indoor security, and organization of indoor services (garbage collection, mail distribution, etc.) are some other areas waiting to be studied. Such applications require network analysis such as shortest path, shortest loop and point to the development of integrated systems including sensorbased communication, control and navigation services (Luo and Wang 2006).

In this study, A robot prototype that can move autonomously without requiring human power and reach a predetermined target on the cardboard platform prepared as a working area has been designed. AlphaBot robot development kit was preferred as the main body (AlphaBot 2021). Because it has enough socket outputs and works with Arduino and Raspberry Pi.

People are prohibited from entering buildings that are in danger of collapse due to disasters such as earthquakes, floods, and fires. However, in these buildings, valuable items and even living things such as babies and pets can be forgotten in the building. The aim of this study is to develop an autonomy or semi-autonomy prototype and a preliminary study for a robot design that can be sent inside the building and transport the things that need to be transported outside of the building in cases like the above.

\section{LITERATURE REVIEW}

In the study called "Indoor Mobile Robot Navigation and Control", an autonomous mobile robot that follows a line has been designed. The controller structure is two-stage. This has been helpful in getting better performance. Ziegler-Nichols Method was used for PID controller and parameters. (Korkut 2013)

In the study called "A Reaction Obstacle Avoidance Algorithm for Autonomous Vehicles", an obstacle avoidance algorithm is designed. The Collision cone method, which has the logic of going around an encountered obstacle, is used. In this study, this method is aimed not only at predetermined obstacles, but also overcoming obstacles encountered in motion (Yucel 2012).

In the study called "Basic Data Communication and Control Infrastructure Design in a Service Robot", a robot design that can follow people and moving objects in any environment has been made. First, the robot's control and data communication infrastructure has been established. Then, tests were carried out using simulation programs. In the testing phase, MINI-ITX motherboard was used (Sar1 2013).

In the study called "Differential Driven Wheeled Robot Design and Implementation for Search and Rescue", a search and rescue themed robot was designed. Designs on ROS were 
carried out practically and experiments were carried out. It has a 4-wheel drive system and each wheel can receive a different speed command. Laser distance sensor is used for position and posture information. For mapping, gMapping is emphasized (Cakmak 2014).

In the study called "Autonomous Robot and Control Unit Design", an autonomous design was made for use in different areas. In this design, LPC1769 is used as a microcontroller. The robot is mounted on a 6-wheel system. UART communication is used for communication (Durmus 2015).

In the study called "An Application for Tracking the Route of Autonomous Vehicles", an application has been made for the route tracking of autonomous vehicles. As a result of this application, it is aimed to reach the route starting from the starting point with the depth priority search algorithm on a predetermined map (Nikbay 2015).

In the study called "Design and Control of an Autonomous Electrical Vehicle for Indoor Transport Applications", a robot that can move autonomously in an indoor area with pedestrians was designed. Detection of pedestrians and estimation of the points where they can move were made by the LIDAR sensor. The reason for this is that the LIDAR sensor gives much more stable results (Gelbal 2015).

In the study called "Position Control of an Autonomous Robot with a Fuzzy Controller Approach", the position control of an autonomous robot was carried out on a fixed track. Hardware control is provided with Atmel 2560 Integration. This controller is programmed with $\mathrm{C \#}$. The robot is given the information of the note it will go on the track and the robot reaches the target point according to the location information. The initial location information was obtained using the Bluetooth module (Cetinkaya 2017).

In the study called "Mobile Robot Development for Educational and Research Purposes", a mobile robot platform that can work indoors was designed. A kinematic and dynamic model has also been prepared for the $3 \mathrm{D}$ simulation environment. Before the tests, remote control was performed. Then the random wandering behaviour was tested, and finally, tests were made by combining both and the system was checked (Akcakoca 2017).

In the study called "RFID Based Positioning Application with Deep Learning", communication between RFID receiver and transmitter was listened. This listening process was done with an external hardware. This data was then used to find the location (Guler 2018).

In the study called "Design and Construction of a Low Cost GPS Based Autonomous Unmanned Ground Vehicle", GPS module and electronic compass sensor are used to determine the location of the vehicle. PIC18f452 microcontroller was used as a decision maker. It has been ensured that the vehicle goes from its current location to the target location autonomously (Hulako and Kapucu 2018).

In the study called "Design and Implementation of an Autonomous Robot for Industrial Purposes", an industrial autonomous or remotely controlled robot design was made using Raspberry Pi and Arduino. Arduino board controls 4 motors. And with the ROS library in it, it is provided to communicate with the ROS program running within the Raspberry Pi. Wheels that can rotate in all directions are preferred and mobility is increased. This makes it easier to avoid obstacles. (Ispir 2019)

In the study called "Embedded System-Based Indoor Autonomous Robot", a robot design that can go to a location specified by the users in the building has been made. Raspberry $\mathrm{Pi}$ is used for robot control. With a camera module and the signs placed at the target points, the robot is provided to go to the specified location. The image was taken using the OpenCV library. As a method, the deep learning method was tried first, but the system load was too high. Instead, the system load is reduced and the response time is shortened by using the Cloud Vision API (Doğan 2019).

In the study called "Embedded System Design for Mobile Robots", a software platform for mobile robots has been developed. It uses the ROS infrastructure and runs on the Linux operating system. An ARM-based controller is used and a depth camera is connected to this board. The information received from the camera is sent to the host via the Wi-Fi module. In this way, the obstacles in front of the robot were determined and it was tried to direct the robot with the necessary angle to avoid the obstacles (Yalçın 2019).

\section{METHODOLOGY}

\subsection{Arduino Based Robot Prototype Determination and Development}

First, the hardware design of the robot was emphasized AlphaBot robot development kit was preferred as the main body (AlphaBot 2021). It has been preferred to have enough socket outputs on it and to work with Arduino and Raspberry Pi. Some modules and sensors such as the assembly of the main body of the robot, the integration of the charging unit, WiFi and RFID reader modules, gyro and Ultrasonic Distance sensors have been added to the development kit (Figure 1).

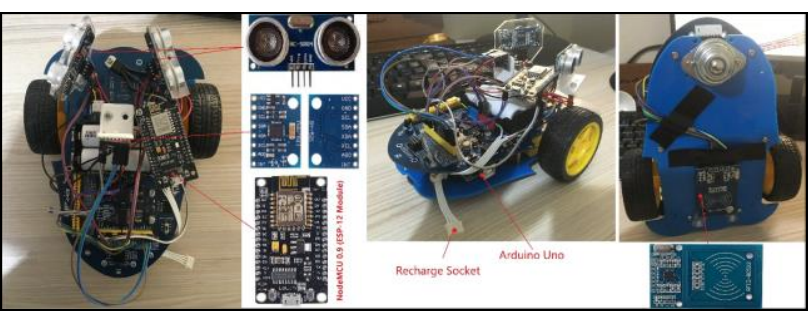

Figure 1. Prototype, Modules and Sensors on it

The list of sensors and modules on the prototype is as follows:

1. Arduino Uno

2. RFID Reader Module (RF522)

3. WiFi Module (Nodemcu 0.9)

4. Gyro Module (GY521)

5. Wheel Speed Sensor (LM393)

6. Ultrasonic Distance Sensor (HC-SR01)

7. Battery Charger Module

Arduino Uno, Robot prototype was used to manage all connected sensors and modules on the circuit. The realization of the routing instructions coming from the $\mathrm{WiFi}$ module, the processing of the data from the distance sensors, the rotation and advancement movements of the prototype and the codes recorded in the Arduino are carried out. The coding was coded with the $\mathrm{C}++$ programming language using the Arduino IDE. 
With the RFID Reader Module, the ID numbers of the RFID cards are read. It has been used for the study, the details of which are given in the next section on RFID-based location determination.

The Gyro Module is considered necessary to calculate the rotation angles. It has been added to the prototype to minimize the error in angular turns by using it with the Speed Sensor.

The Wheel Speed Sensor is available on the AlphaBot kit. It prevents situations such as decrease or increase in wheel rotation speed depending on the charge rate of the battery. It offers the possibility to determine the speed using software. In addition, it allows the wheels to rotate independently of each other.

The WiFi Module is used to transfer the routing instructions sent from the server to the Arduino.

Ultrasonic Distance Sensor is used to prevent the prototype from going through the middle of the path and hitting the obstacles that may come in front of it during the movement.

Battery Charger Module, Prototype has two 18650 batteries connected in series for power supply with $7.4 \mathrm{~V}$ input voltage. The cables that will connect the B3 pro compact charger were soldered to the battery compartment where the battery was inserted to facilitate the charging process of the batteries,

\subsection{Creation of the Test Area and Installation of the RFID Based Positioning System}

At this stage, a test area of $80 \mathrm{~cm}$ x $80 \mathrm{~cm}$ was created using cardboard material as shown in Figure 2. RFID cards for the positioning system are placed on the cardboard plate. Our experience with the RFID-based positioning system for indoor spaces, which we developed in our previous work together with some of our teammates in our 3DGIRG (3D Geo-Informatics Research Group) working group, was utilized in this study (Demiral et al., 2013), (Karas et al., 2017), (Demiral et al.,2014).

The test area is divided into square areas as shown in Figure 2. The size of each of the square areas is designed as $20 \mathrm{~cm} \times 20$ $\mathrm{cm}$. RFID cards representing square areas at the midpoint of the square areas are shown in Figure 2 on right side. However, as it can be seen in Figure 1, considering the location of the RC522 RFID reader on the Alphabot Kit (prototype), the RFID cards are not placed in the middle of the square areas, but are placed on the edges of the square sections, as in the image of the test area made of cardboard plate in the image on the left in Figure 2.

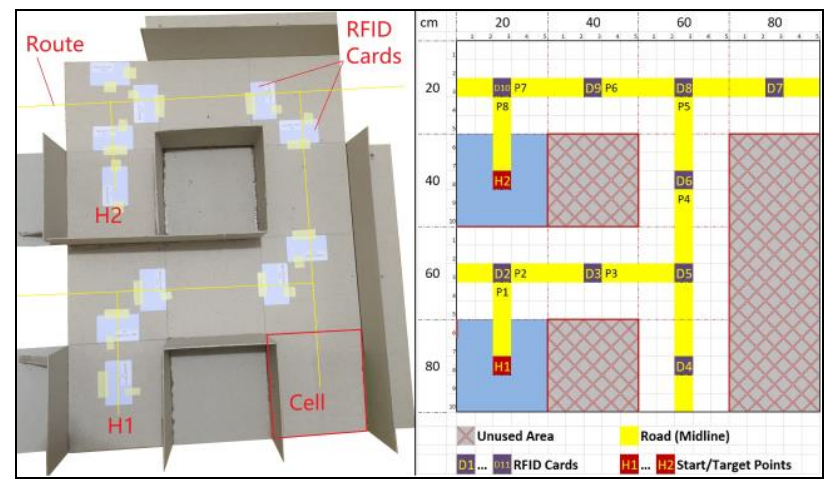

Figure 2. Test Area
No RFID cards are placed in the square areas where the prototype goes forward to next square directly. There are RFID cards in the square areas where the prototype needs new rotation command such as turn left or right or forward to reach the next square area. When the prototype enters a square area, it needs to turn, it reads the RFID card and stops. It sends the ID number of the card it reads to the server. On the server side, the next routing command is determined by considering the instantaneous location of the prototype. Afterwards, the prototype performs its next move according to the routing command sent from the server.

The yellow line shows the paths the prototype can move. There are two different target points. Also, RFID cards are located at these target points. When the prototype reaches its destination, it reads the RFID card placed in the square area and sends the ID number of this card to the server. When this information reaches the server, the user is presented with the "Target Point Reached" information. The prototype waits at this point until a new target is determined by the user.

\subsection{Detecting the Position of the Robot}

At this stage, RFID cards placed on the cardboard platform are used. The RF522 RFID reader integrated on the prototype is a low frequency and close-range reader. The reading distance is up to $5 \mathrm{~cm}$. While moving on the prototype platform, it passes over the RFID cards on the route. The RFID reader placed under the robot reads the ID number of the card during these passes. The location of each RFID card in the working area is recorded on the server. The square areas in which the prototype needs new rotation command are considered. No RFID card is inserted for square that are direct passage and do not have any return status. Therefore, the read ID number means that the prototype is within a square area that needs to be rotated. According to this information, the routing of the mentioned prototype is carried out given details in the next stage of the study. If the current location contains alternative turns, the prototype will wait at this location until the redirect command comes from the server via WiFi protocol. The response time from the server may take a few seconds. According to the sequential routing commands send from the server, the prototype moves until it reaches the target. The ID numbers of the RFID cards placed in the square areas determined as the target were labelled differently from the others and recorded as "Target Point".

RFID cards can also be placed in square areas where the prototype goes forward to next square directly, and users can be presented with the instant location of the prototype in more detail. However, this information was not needed in this study due to the small test area.

\subsection{Development of Network Analysis Module and Instruction Module to Direct the Robot}

After the stage of determining the location of the robot, the stage of directing the robot to the target comes. At this stage, first, the starting and destination points of the robot must be determined. The starting point is accepted as the current position of the robot. The destination will be one of the destinations to be selected by the user. After these two critical points are determined, the software determines the shortest path between the two points, that is the movement route. In the preparation of the network analysis module, a similar network analysis and navigation study that we developed as the 3DGIRG 
working group in our previous work for person routing was utilized (Ortakci et al, 2014) and (Ortakci et al, 2016).

Routing instructions are sent to the prototype via the WiFi module according to the movement route determined by the server. When a new RFID card is read by the RFID reader on the prototype, the ID number information is also transmitted from the prototype to the server. On the server side, the location information matching the incoming ID number is checked. If the target point was not reached, the instruction to move to the next square is transmitted to the prototype. For example, turn right and move forward. Routing processes continue until the prototype arrives at the destination. When the prototype arrives at the target, a notification message is shown to the user on the server side that the prototype has reached the target, and the prototype waits at this point until a new target information is entered.

\section{SYSTEM IMPROVEMENT}

In this study, AlphaBot was used as a prototype. Alphabot is a robotics development platform compatible with Raspberry Pi and Arduino. It consists of the motherboard, the mobile case and the materials needed to move it. Raspberry Pi / Arduino interfaces can work with either one or both. Plug and play modules such as modular design, line monitoring, obstacle avoidance, speed measurement eliminates the hassle of connecting tangled cables (AlphaBot 2021). Arduino was used as the microprocessor. Coding is also done on Arduino IDE.

The sensors are LM393 speed sensor, RFID card reader, distance sensors and WiFi communication module are used. With the LM393 speed sensor, it is possible to calculate the distance travelled by the wheels. Based on this information, the angle of rotation was found. However, here, factors such as the filling rate of the battery, the fact that the wheels are not identical even with small differences, and the friction coefficient of the floor, the slipping of the wheels during the first movement or stopping can cause angular deviations. This is an important issue that needs to be mentioned. Angular deviation appears to be a problem as it affects the next direction of motion. As a solution to this problem, barriers placed on the sides of the movement paths on the cardboard plate were used. By processing data that comes from the distance sensors using PID controller, it is ensured that the prototype avoids the obstacles on the cardboard plate on the sides of the roads and goes to middle of the road it is on. The RFID reader is used to detect the position of the prototype, and the WiFi card is used to communicate between the prototype and the server. When the RFID reader on the prototype reads a new card, the RFID card ID is sent to the server via the WiFi card integrated on the Arduino. Based on this information, the routing instruction generated on the server side is also transmitted from the server to the prototype via the WiFi card.

\subsection{Rotation Angle Calculation Using Speed Sensor}

For this process, first, LM393 speed sensor and encoder disks were connected to each motor. The wheel and encoder disc are connected to the same shaft (Figure 3). So, the speed of both will be equal. In other words, it was sufficient for us to measure the speed of the encoder disk to find the speed of the wheel. As it is known, there are gaps on the encoder disk. These gaps are counted by the speed sensor as the shaft rotates, and thus the speed information is reached. At the same time, the distance travelled can be measured thanks to the speed information and the known radius of the wheel. By applying this process to the other wheel, angle information was obtained without using the gyro sensor. To make a note here, various attempts have been made to obtain angle information using the Gyro sensor, but it is not covered in this study because of insufficient success.

For the angle value, rotation of the prototype to the left is taken as a positive angle, and the rotation to the right is taken as a negative angle. For the wheel to make one full revolution, the space on the decoder disk must be counted 40 times from the speed sensor. For example, if this number is read for the right wheel, it means that the right wheel has made one full turn, which will mean that the prototype rotates to the left at a 90 degrees angle. This is the same for the other wheel. In the initial state, the angle value is accepted as 0 . In this case, the prototype will continue straight ahead. The instantaneous angle value is found by subtracting the left wheel angle from the right wheel angle.

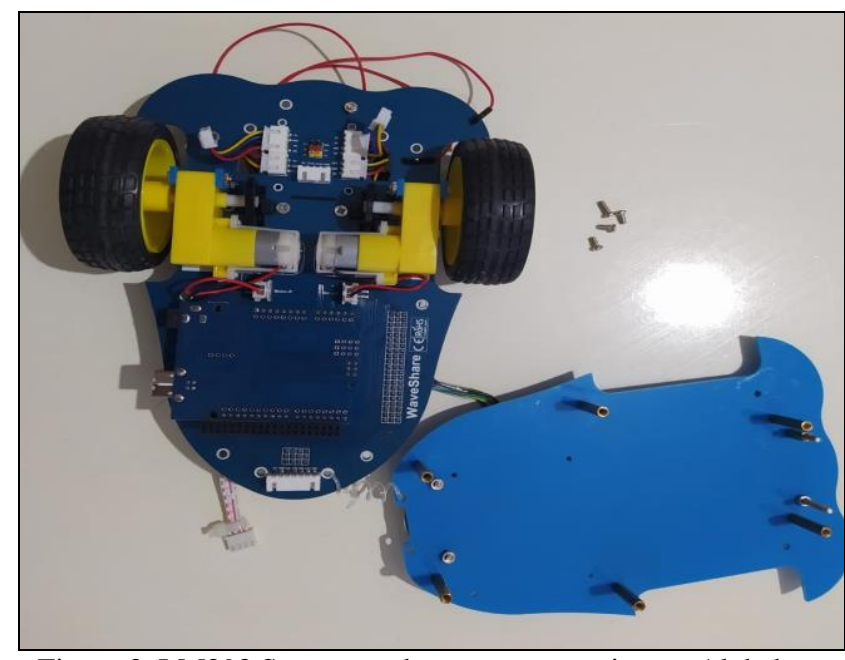

Figure 3. LM393 Sensors and motors connection on Alphabot

$$
r p m=(1000 / \text { time taken }) * 60,
$$

To find the speed value:

$$
\text { Speed }=2 \pi * r p m *(\text { radius of wheel }),
$$

Left wheel angle $=(360 \%$ number of cuts $) *(90 / 40)$,

$$
\text { Right wheel angle }=(360 \% \text { of cuts }) *(90 / 40),
$$

$$
\text { Instant angle }=\text { right wheel angle }- \text { left wheel angle, }
$$

After the angle information is reached, the wheel in the direction of rotation must remain stationary and the wheel on the opposite side must rotate for the vehicle to turn. The distance travelled by the wheel that will move here is determined depending on the angle value. 


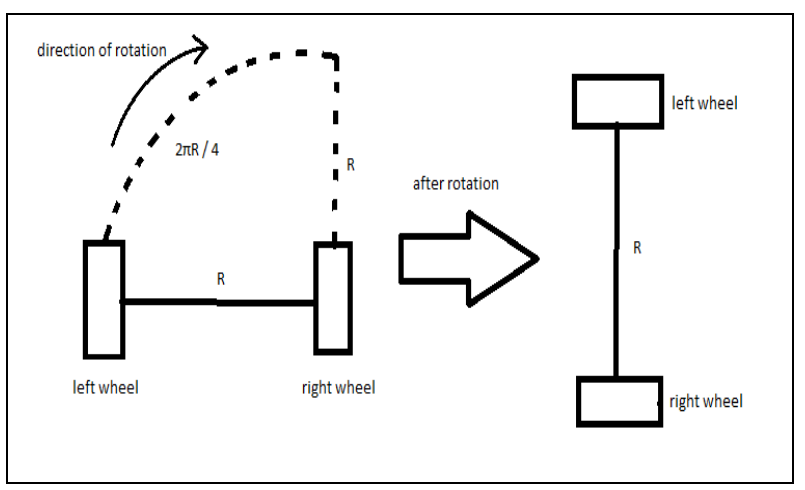

Figure 4. Right turn, In order to turn right, the right wheel remained stationary, the left wheel travelled $2 \pi \mathrm{R} / 4$ and completed the 90 degrees turn

\section{CONCLUSIONS}

In this study, Subject of Design of robot prototype for indoors guided by RFID Based Positioning and Navigation System is examined. Within the scope of the study, a prototype robot was made. It was carried out determine the current position of the robot with the positioning system and to send it to the target determined by the user autonomously on cardboard platform. In this context, with the developed software and modules, the robot was tested in the test area and it was observed that it reached the target. It is foreseen that better results can be obtained in future studies if the problems encountered during the determination of rotation angles can be eliminated by using different methods and sensors. Considered in this context, it is a promising study.

\section{ACKNOWLEDGEMENTS}

This study has been supported by YÖK (Council of Higher Education in Turkey) (Project No: MEV.2018-1342). We are indebted to their supports.

\section{REFERENCES}

Ko, A. W. Y. and Lau, H. Y. K., 2009: Intelligent RobotAssisted Humanitarian Search and Rescue System. International Journal of Advanced Robotic Systems, doi: $10.5772 / 6792$.

R. C. Luo, P. K. Wang, Y. F. Tseng and T. Y. Lin, 2006: Navigation and Mobile Security System of Home Security Robot. IEEE International Conference on Systems, Man and Cybernetics, pp. 169-174, doi: 10.1109/ICSMC.2006.384377.

AlphaBot. AlphaBot - Waveshare Wiki. Available at: https://www.waveshare.com/wiki/AlphaBot. Accessed July 8, 2021.

Korkut, O. Indoor Mobile Robot Navigation and Control Master thesis, Dokuz Eylul University Institute of Science and Technology, Izmir, Turkey (2013)

Hulako, $\mathrm{H}$ and Kapucu, S Design and Construction of a Low Cost GPS Based Autonomous Unmanned Ground Vehicle Gazi University Journal of Science PART C: DESIGN AND TECHNOLOGY GU J Sci, Part C, 6(4): 834-850 (2018)

Ispir, M. T. Design and Implementation of an Autonomous Robot for Industrial Purposes, Master Thesis, Firat University, Institute of Science and Technology, Elazig, Turkey, (2019)
Dogan, R. Embedded System Based Indoor Autonomous Robot Master Thesis, Marmara University, Institute of Science and Technology, Istanbul, Turkey (2019)

Sari, A.Ç. Design of Basic Data Communication and Control Infrastructure in a Service Robot Master Thesis, Istanbul Technical University, Institute of Science, Istanbul, Turkey (2013)

Cakmak, F. Differential Driven Wheeled Robot Design and Implementation for Search and Rescue Purposes Master Thesis, Ylldiz Technical University, Institute of Natural sciences Istanbul, Turkey (2014)

Durmus, H. Autonomous Robot and Control Unit Design Master Thesis, Istanbul Technical University, Institute of Natural sciences, Istanbul, Turkey (2015)

Cetinkaya, A. "Position Control of an Autonomous Robot with Fuzzy Controller Approach" Master Thesis, KTO Karatay University Institute of Science and Technology, Konya Turkey (2017)

Akcakoca, M. Development of Mobile Robot for Educational and Research Purposes Master Thesis, Eskisehir Osmangazi University Institute of Science and Technology, Eskisehir Turkey (2017)

Yalcin, H. S. Embedded System Design for Mobile Robots Master Thesis, Firat University Institute of Science and Technology, Elazig, Turkey (2019)

Guler, S. RFID Based Positioning Application with Deep Learning Master Thesis, KTO Karatay University Institute of Science and Technology, Konya Turkey (2018)

Nikbay, K. An Application for Route Tracking of Autonomous Vehicles Master Thesis, Okan University, Institute of Science and Technology, Istanbul, Turkey (2015)

Gelbal, Ş.Y. Design and Control of an Autonomous Electrical Vehicle for Indoor Transport Applications. M.Sc. Thesis, Istanbul Technical University Graduate School of Science Engineering and Technology Istanbul, Turkey (2015)

Yücel, G. A Reaction Obstacle Avoidance Algorithm for Autonomous Vehicles M.Sc. Thesis, Middle East Technical University, Ankara, Turkey (2012)

Demiral E., Karaş İ.R., Turan M.K., "RFID Sistemleri ile Konum Belirleme Uygulamaları", 14. Türkiye Harita Bilimsel ve Teknik Kurultayl, Ankara, Türkiye (2013)

Karas I.R., Atila U., Demiral E. "Developing a 3D Routing Instruction Engine for Indoor Environment". In: Yuan H., Geng J., Bian F. (eds), Pages: 34-42, Geo-Spatial Knowledge and Intelligence. Communications in Computer and Information Science (CCIS), vol 698. Published by Springer, Singapore, ISBN 978-981-10-3965-2 (2017)

Emrullah Demiral, Ismail Rakip Karas, Muhammed Kamil Turan, Umit Atila, "RFID-based mobile positioning and navigation system design for 3D indoor environment", 9th International 3D Geoinformation Conference (3DGeoInfo'14), Dubai, United Arab Emirates (2014)

Yasin Ortakci, Emrullah Demiral and Ismail Rakip Karas, "RFID Based 3D Indoor Navigation System Integrated With 
Smart Phones", 11th International Symposium on Location Based Services, Vienna, Austria (2014)

Yasin Ortakc1, Umit Atila, Emrullah Demiral, Ismail Rakip Karas, "Intelligent Mobile Indoor Navigation System for Fire Evacuation Based on Artificial Neural Network", International Journal of Computer Science and Information Security, Vol 14 (11), pp. 980-996 (2016)

3DGIRG - 3D Geo-Informatics Research Group. Members of Research Group: https://3dgirg.karabuk.edu.tr/members.htm Accessed July 8, 2021. 